\title{
Muerte por sumersión debida a shallow water blackout
}

\section{Death due to drowning by shallow water blackout}

\section{Resumen}

El llamado shallow water blackout, o síncope de las aguas superficiales, es un accidente que pueden sufrir los buceadores y llevarles a la muerte por sumersión. La natación sumergido (buceando) precedida de hiperventilación crea una situación en la que el sujeto puede sufrir hipoxia antes de que la concentración en sangre arterial de dióxido de carbono alcance el nivel que le obligue a salir a la superficie a respirar. En esta situación, el sujeto inconsciente puede respirar bajo el agua y morir por sumersión.

Palabras clave: Shallow water blackout (síncope de las aguas superficiales). Buceo en apnea. Hipocapnia por hiperventilación. Hipoxia. Sumersión.

\section{Abstract \\ We report a case of drowning due to shallow water blackout. This happens when divers deliberately hyperventilate and then enters the water to see how long they can stay submerged or how far they can swim underwater. In this situation the $\mathrm{PaO} 2$ may fall to a level sufficient to cause loss of consciousness before de $\mathrm{PaCO} 2$ has risen enough to trigger ventilation. Once they lose consciousness they drive to breathe and inhale water, thus initiating the drowning process.}

Key words: Shallow water blackout. Breath holding dive. Swimming underwater. Hyperventilation hypocapnia. Hypoxia. Drowning.

\section{Introducción}

Desde hace muchos años se conoce un accidente que pueden sufrir los buceadores y que puede llevarles a la muerte por sumersión, especialmente si no están advertidos y se encuentran solos en el momento de sufrir dicho accidente.

Sobre el trastorno existen muchas publicaciones en la literatura médica desde hace varias décadas ${ }^{1,2}$ y pocas, aunque algunas, en la médico-legal ${ }^{3}$, con el nombre de shallow water blackout, síncope o pérdida de consciencia en aguas superficiales. Hasta el año 2006 no se encuentra ninguna referencia en un prestigioso texto de patología forense ${ }^{4}$.

Cuando se practica el buceo en apnea ("a pulmón libre") es bien conocido que hiperventilar antes de sumergirse puede prolongar el tiempo de inmersión. Con la hiperventilación se consigue iniciar la apnea en hipocapnia, y de esta forma, la concentración de dióxido de carbono que estimula al centro respiratorio hasta el punto de que el sujeto ya no puede inhibir voluntariamente la respiración (el llamado breaking point o punto de ruptura de la apnea $)^{5}$ y tiene que salir a la superficie a respirar, tarda algo más en presentarse.

El accidente se origina cuando el sujeto hiperventila excesivamente antes de la inmersión y hace un gran esfuerzo al nadar sumergido. Entonces puede ocurrir que, antes de que la concentración de dióxido de carbono alcance el nivel que le obligue a salir a la superficie a respirar, se produzca una hipoxia que le ocasione al sujeto una pérdida de la consciencia $y$, en este estado, respire bajo el agua y muera por sumersión, salvo que sea rescatado en los primeros momentos. En los diagramas de las Figuras 1 y 2 se representan las evoluciones de los gases en sangre arterial, oxígeno y dióxido de carbono, durante la

\section{JL. Palomo Rando ${ }^{1}$ \\ V. Ramos Medina ${ }^{1}$ MA. Calvo López ${ }^{1}$ IM. Santos Amaya²}

${ }^{1}$ Médico Forense. Instituto de Medicina Legal de Málaga

2Profesor del Área de Medicina Legal y Forense. Universidad de Málaga.

\section{Correspondencia:}

José Luis Palomo Rando Instituto de Medicina Legal Ciudad de la Justicia Fiscal Luis Potero, 6 29010 Málaga

E-mail: jlpalomo45@telefonica.net

Fecha de recepción: 10. OCT. 2014

Fecha de aceptación: 14. NOV. 2014 
Figura 1.

Buceo en apnea tras la respiración normal. Después de iniciar el buceo, cuando el nivel de $\mathrm{CO}_{2}$ en sangre arterial alcanza una concentración con la cual la respiración no puede ser inhibida voluntariamente (el llamado breaking point o punto de ruptura de la apnea), el sujeto se ve obligado a salir a la superficie a respirar. La presión de $\mathrm{CO}_{2}$ en dera que produce un estimulo muy intenso de la respiración es de $60 \mathrm{mmHg}^{10}$. sangre arterial que se consi-

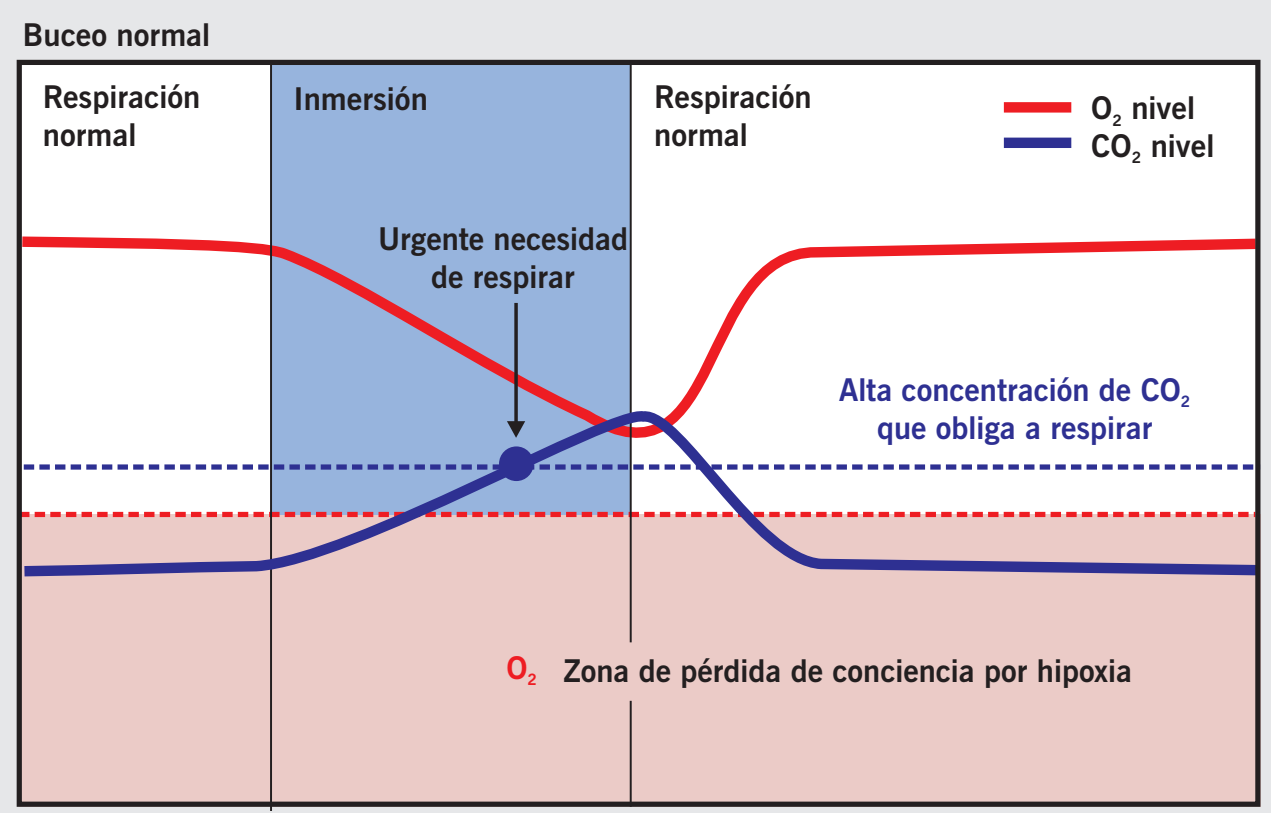

Figura 2.

Buceo en apnea con hipocapnia, tras hiperventilación. En este caso la hipoxia, que impide el normal funcionamiento del cerebro y puede hacer perder la consciencia, se presenta antes de que el nivel de

$\mathrm{CO}_{2}$ llegue al breaking point que obligaría al sujeto a salir a la superficie a respirar. Esta es la causa de/ shallow water blackout, en el que el sujeto inconsciente respira bajo el agua y se ahoga. Una presión de $\mathrm{O}_{2}$ en sangre arterial de $34 \mathrm{mmHg}$ o menos se asocia generalmente con pérdida de consciencia si se instaura bruscamente?

\section{Buceo en Hipocapnia}

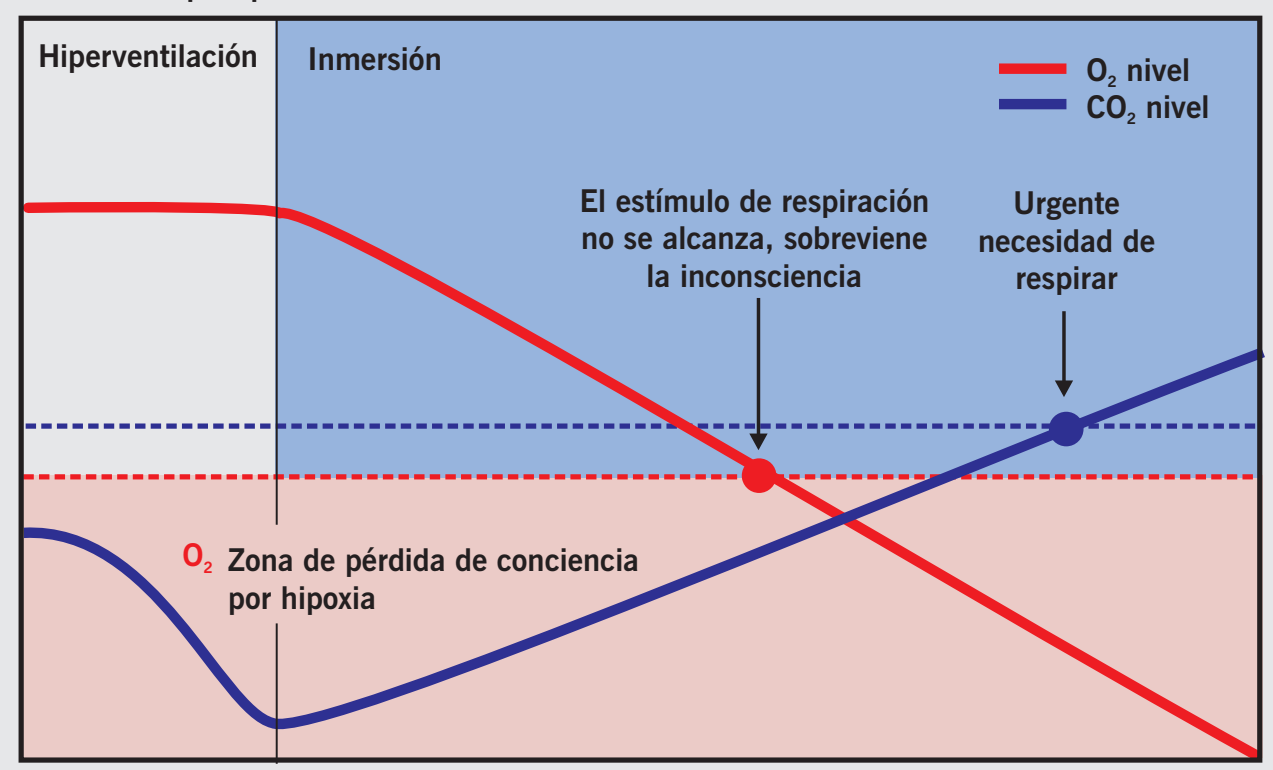


inmersión en condiciones normales y en hipocapnia por hiperventilación previa.

Este accidente no debe confundirse con otro que, también por hipoxia, pueden sufrir los buceadores en apnea cuando descienden más de 7-10 metros. Este otro tipo de accidente se debe esencialmente a que al ascender disminuye la presión hidrostática y, en consecuencia, la presión parcial de oxígeno. Probablemente por este mecanismo fallecen por sumersión algunos practicantes de pesca submarina en nuestro país. Este evento es más probable y grave si también el sujeto hiperventila antes de la inmersión.

\section{Presentación del caso}

Varón de 20 años de edad, ciudadano de un país escandinavo, sin antecedentes patológicos, que falleció en la piscina del hotel donde se alojaba en una localidad turística española. El ahora fallecido, que pasaba unos días como turista en el hotel, se encontraba preparándose para ingresar como profesional en el ejército de su país. Una de las pruebas físicas que tendría que superar consistía en nadar bajo el agua (bucear) una determinada distancia, lo que intentaba todos los días y para lo que tenía bastante dificultad en conseguir su objetivo. Su familia informó al médico forense que acudió al levantamiento que constituía para él una obsesión poder alcanzar dicha distancia y que llevaba un registro diario de los metros conseguidos. Aprovechaba las últimas horas de la tarde, cuando no había nadie en la piscina, para entrenarse. Fue visto nadando y posteriormente advirtieron que estaba en el fondo de la piscina en decúbito prono sin reaccionar. Asistió el servicio de emergencias sanitarias, que lo encontró en parada cardiorrespiratoria y aplicó medidas de reanimación sin éxito.

En la autopsia cabe destacar hongo de espuma a pesar de la prolongada reanimación avanzada a la que fue sometido, intenso edema pulmonar confirmado histopatológicamente como compatible con sumersión, y signo de Niles, aspecto hemorrágico del peñasco del temporal; todos ellos signos inespecíficos. El corazón, las coronarias y el resto de los órganos eran normales macroscópicamente y microscópicamente. La investigación de alcohol y de tóxicos en sangre y orina fue negativa.

La muerte se estableció como de origen violento y su causa como sumersión. En la valoración se consideró que el mecanismo más probable que ocasionó la sumersión fue el shallow water blackout.

\section{Discusión}

La producción de este fenómeno (shallow water blackout) es indemostrable en la autopsia, y su existencia es conocida por los numerosos casos ocurridos y publicados en la literatura médica y médico legal. El testimonio de los que fueron rescatados y sobrevivieron al accidente fue lo que puso en marcha las investigaciones ${ }^{2}$.

Los diagnósticos en patología forense, como en cualquier otra especialidad médica, pocas veces son de absoluta certeza, e incluso cuando hay lesiones traumáticas incompatibles con la vida cabe plantearse si estas pudieron haberse producido post mórtem. En muchos casos son esenciales para el diagnóstico los antecedentes y las circunstancias que rodearon y condujeron a la muerte. Un caso paradigmático es la electrocución por electricidad doméstica con ausencia de marca eléctrica, en el cual la autopsia es "blanca" o negativa. En estos casos, el diagnóstico tiene que basarse en el examen de la escena y sobre todo de los dispositivos eléctricos existentes en ella, tanto o más que en la propia autopsia.

Por ello, en muchos casos el diagnóstico de la causa de la muerte tras la autopsia judicial, cuando no existen pruebas definitivas, es un diagnóstico de exclusión (entre ellos está el diagnóstico de la propia sumersión): después de conocer los resultados de las pruebas complementarias indicadas y los antecedentes y las circunstancias de la muerte, y descartadas otras causas menos probables, se establece el diagnóstico de la causa más probable.

En este caso, el diagnóstico de shallow water blackout como mecanismo causante de la sumersión se basó en que era un sujeto sin antecedentes patológicos de interés, personales ni familiares, a los que atribuir un ahogamiento inesperado (no epilepsia ni convulsiones, no casos de muerte súbita en familiares jóvenes, etc.), muy entrenado como nadador, que se encontraba solo en una piscina de 2,5 metros de profundidad máxima y en cuya autopsia no se encontraron otras alteraciones morfológicas distintas de la sumersión ni presencia de tóxicos, y sobre todo el antecedente de la patente dificultad e insistencia del sujeto para conseguir el tiempo de apnea requerido en las pruebas que estaba preparando.

En libros y revistas de patología forense hay pocas descripciones de este mecanismo como causa de muerte por sumersión. Sin embargo, existen muchas publicaciones médicas que comunican casos de 
muerte por este mecanismo y la necesidad de que la población conozca el peligro de la hiperventilación antes de sumergirse para bucear ${ }^{6}$. El accidente ocurre casi siempre en varones con una edad media entre 16 y 20 años, generalmente buenos buceadores y nadadores, y el $80 \%$ de los casos en piscinas vigiladas. Más de la mitad han sobrevivido al accidente al ser rescatados precozmente. Por ello se insiste en la necesidad de no practicar el buceo en solitario ${ }^{7}$. En numerosas páginas web de entidades dedicadas al buceo, incluida una de la Marina de los Estados Unidos, se advierte insistentemente a los buceadores del peligro de hiperventilar excesivamente antes de la inmersión ${ }^{8,9}$, lo que ha costado la vida a muchas personas.

\section{Conclusión}

Aunque en nuestro país no está muy extendida la práctica del buceo, y en la mayoría de los casos comunicados los accidentes ocurren en el buceo con escafandras automáticas (SCUBA, self contained underwater breathing apparatus), es conveniente dar a conocer este fenómeno entre los médicos forenses, pues es un hecho que puede explicar la serie de acontecimientos que conducen a algunas muertes por sumersión accidental, que de otra forma se considerarían inexplicables.

Los autores declaran no tener ningún conflicto de intereses.

\section{Bibliografía}

1. Craig $A B$ Jr. Underwater swimming and loss of consciousness. JAMA. 1961;176:255-8.

2. Craig BA Jr. Causes of loss of consciousness during underwater swimming. J Appl Physiol. 1961;16: 583-6.

3. Davis JH. Fatal underwater breath holding in trained swimmers. J Forensic Sci. 1961;6(3):301-6.

4. Spitz DJ. Investigation of bodies in water. En: Spitz WU, Spitz DJ, editores. Spitz and Fisher's Medicolegal investigation of death: guidelines for the application of pathology to crime investigation. 4th ed. Springfield, IL: Charles C. Thomas; 2006. p. 849.

5. García-Cosío Mir F (coordinador), Alberca Vela T, Rubio Sanz J, et al. Guías de práctica clínica de la Sociedad Española de Cardiología sobre conducción de vehículos, pilotaje de aviones y actividades subacuáticas en cardiópatas. Rev Esp Cardiol. 2001;54:476-90. (Consultado el 05-09-2014.)
Disponible en: http://www.revespcardiol.org/es/ guias-practica-clinica-sociedad-espanola/articulo/11000059/.

6. Modell JH. Prevention of needless deaths from drowning. South Med J. 2010;103(7):650-3.

7. Craig AB Jr. Summary of 58 cases of loss of consciousness during underwater swimming and diving. Med Sci Sports. 1976;8(3):171-5.

8. Naval Safety Center. Official U.S. Navy Web Site. (Consultado el 15-09-2014.) Disponible en: http:// web.archive.org/web/20080214122244/http:// www.safetycenter.navy.mil/articles/shallowwater.htm

9. Dive Wise. (Consultado el 17-09-2014.) Disponible en: http://divewise.org/education/freediver-blackout/

10. Estados confusionales agudos y coma. En: Harrison. Principios de medicina interna. 16a ed. México: McGraw-Hill Interamericana; 2006. p. 1.800. 